\title{
An Application of Restricted Open Queueing Networks to Healthcare Systems
}

\author{
M. S. Sreekala \\ Department of Statistics, \\ University of Calicut, Kerala, India. \\ M. Manoharan \\ Department of Statistics, \\ University of Calicut, Kerala, India.
}

\begin{abstract}
In this paper we discuss some of the crucial aspects of open restricted queueing networks with special emphasis on the issues of limitations on the capacity of the service provided as well as on the accommodations of the customers. We review the literature bringing together all relevant materials in this problem area. Here we develop the expansion method for approximating the flow process of customers as they travel through this restricted system. Further we present numerical experiments illustrating the proposed method and conduct a comparative study.
\end{abstract}

Keywords - Queueing networks, restricted network, blocking probability, healthcare system.

\section{INTRODUCTION}

Queues are formed when entities that demand service, usually referred to as 'customers', arrive at a 'service facility' and cannot be served immediately upon arrival. In healthcare delivery systems, patients are typically the customers and either outpatient clinics or hospitals or diagnostic imaging centers are the service facilities. A service facility may consist of one or more service stations where customers are served. Following Bose [2], a queueing network is a system of interconnected queues providing service. Queueing networks can be classified as open, closed and mixed with respect to the number of customers in the network. In an open network customers enter from outside, receive service at systems and leave the network. In closed network, a constant number of customers continually circulate in the network with no other arrival to the system and departures from the system. Depending on the number of customer classes we have single or multi class networks. In the case of mixed network, the network may be open for some classes of customers and closed for some other classes. Queueing network models have various applications in many areas, such as service centers, computer networks, communication networks, production and flexible manufacturing systems, airport terminals and health-care systems etc. Most of these physical systems have finite capacity; the customers receiving service is restricted. Such systems are more complex but widely applicable.

In a previous paper Sreekala and Manoharan [29] have reported the use of decomposition method for the analysis of a healthcare system of infertility clinic considered as an open queueing network with blocking. In this paper we are modeling a healthcare situation as a restricted open finite queueing network with blocking and feedbacks. In the case of finite queueing networks, the flow of customers in the system is dependent on the availability of space at the buffers of the downstream nodes. This situation lead to blocking and the network do not have a product form solution which is a desirable proposition in any queueing network system. A blocking mechanism is a set of rules specifying a node is blocked and unblocked. Transfer Blocking (Blocking After Service or BAS) is a one of the popular blocking mechanism considered in the literature (see Perros [25]). In transfer blocking a customer can finish their work at the upstream node and then is blocked if there is no room available at the downstream node. The customer remains in the network at the upstream node and is not lost. When there is available space at the downstream node, the blocked customer proceeds to the downstream node. Healthcare systems in general and hospitals in particular, constitute a very important part of the service sector. Over the years, hospitals have become increasingly successful in deploying medical and technical innovations to more effective clinical treatments. However, they are still often rife with inefficiencies and delays, thus presenting a propitious ground for research in numerous scientific fields, specifically queueing theory. Of particular interest to queueing scientists is the topic of patient flows in hospitals; improving it can have a significant impact on quality of care as well as on patient satisfaction. Hence patient flow management is an important topic of the present time and presents itself with a host of challenging and important research questions. Obviously, patient flow has caught the attention of researchers in operations research, applied probability, service engineering and operations management; with queueing theory being a common central theme on all these disciplines. The reason is that hospitals experience frequent congestion which results in significant delays. Hence they fit naturally under 
the framework of queueing theory, which addresses the trade-offs between operational service quality versus resource efficiency. Our attempt here is to stimulate relevant research, with ultimate goal being delay reduction with its accompanying important benefits: clinical, financial, psychological and societal. Healthcare systems offer many complex problems that could benefit from operations research type analysis and applications (see Carter [4]). The long waiting times of patients, the lack of resources and offload delay problems facing the medical services are among these issues.

\section{RESTRICTED QUEUEING NETWORKS AND ANALYTICAL ISSUES}

Because of the restricted queueing systems the exact solutions are rare except for very simple cases. So the approximations and simulation are often required in such studies. The approximation techniques available in the literature to name some important ones are isolation methods, repeated trials, node-by-node decomposition and expansion method. In isolation method, the network is subdivided into smaller networks and studied in isolation (see Labetoulle and Pujolle [22]). Repeated trials method is a class of techniques based upon repeatedly attempting to send blocking customers to a queue causing the blocking (see Fredericks [6]). In node-by-node decomposition, the network is broken into smaller networks with augmented service and arrival patterns and studied separately on the performance of overall network. This is a very popular method introduced by Reiser and Kobayashi [26] and Kuehn [21]. After them several researchers effectively used this approach in queueing networks. The expansion method is a combination of repeated trials and node-by-node decomposition proposed in various situations by Kerbache and MacGregor Smith [18,19], Jain and Smith [16], Kavusturucu and Gupta [17]. Recently Cruz and Van Woensel [5] used this concept for performance evaluation of finite queueing networks. In order to evaluate the performance of a queueing network, an extra node is added in front of every node and blocked customers are repeatedly sent to this node until a space is available at the full buffer. The addition of holding node expands the network and transforms the network into an equivalent Jackson network in which each node can be solved independently.

The movement of feedback in health care system poses greater challenge for the conventional modeling with the queueing network. Though there are some works on queueing network systems with finite capacity there seems to be rather little work re-ported on health care systems with feedback. Our starting point is that a queueing network encapsulates the operational dimensions of patient flows in hospitals, with medical units being the nodes of network; patients are the customers, while beds, medical staff and medical equipments are the servers. One needs to consider the special features of this queueing network in terms of the system primitives, key performance measures and available control mechanisms. Through the extensive data set of patient flow based on the queueing network model, we may get more insight on the whole system. Queueing models have a long tradition of being useful tools for evaluating the performance of healthcare systems in which waiting lists occur, ever since the work of Bailey [1]. For in-stance Jackson et al. [15], Worthington [31] and Goddard et al. [7] modeled appointment systems and waiting list management in outpatient clinics. Patients flow in hospitals has been extensively studied by many researchers. One may refer to the work of Hall [9, 10] for the methodological advancement of patient flow analysis in healthcare. Roumani [27] suggests a queueing network model of ICUs using discrete event simulation, provides network model for patient flow considering both instantaneous and delayed feedback. The most significant contribution in queueing network is Jackson's network (see Jackson [13, 14]) wherein the joint state distribution for the system can be written as the product of individual state probabilities. Indeed it is applicable for an arbitrary network of $\mathrm{M}|\mathrm{M}| \mathrm{m} \mid \infty$ queueing nodes, where jobs arrive in Poisson stream from outside (to one or more stations) and are routed to one node to another until they eventually depart from the system. In the literature there are many attempts to represent a health care system as network of queues. But many of them are analyzed using computer simulation. Taylor and Keown [30] analyze a system model for Burn care. Harper and Shahani [11] suggest a simulation model in the planning and management of bed capacities in hospitals. Hershey et al. [12] suggest a methodology for estimating expected utilization and service level for a class of capacity constrained service network facilities operating in a stochastic environment. Most of the literature in the healthcare system deals with single station queueing systems. Using the result of Mirasol [23] we have that for a queueing system with a Poisson input and an infinite number of channels the output is also Poisson regardless of the distribution of service times, provided these times are independent. Motivated by the application of queueing network in a healthcare system of an infertility clinic we investigate a model for an open queueing network consisting of three $\mathrm{M}|\mathrm{M}| 1$ nodes with immediate and delayed feedback and propose its steady state solution.

\section{STRUCTURE AND ROUTING PATTERNS OF THE SYSTEM}

We consider an infertility clinic as an open queueing network system, where the patients/couples arrive at the clinic according to a homogeneous Poisson process and get self-service with a general service time distribution for completing an initial non-clinical registration formality. According to the revised glossary of the International Commit-tee for Monitoring Assisted Reproductive Technology (ICMART) and the World Health Organization (WHO) the clinical definition of infertility is "a disease of the reproductive system defined by the failure to achieve a clinical pregnancy after twelve months or more of regular unprotected sexual intercourse" 
(see Zegers-Hochschild et.al [32]). For the treatment of this disease the male and female partners are evaluated for infertility or sub fertility using a variety of clinical interventions and also from certain laboratory evaluations on the individuals. At the initial registration facility, specific enquiries about lifestyle and sexual history of couples are collected to identify people who are less likely to conceive. After completing this formality they enter into the system that is the first node which follows an $\mathrm{M}|\mathrm{M}| 1|\mathrm{~K}| \mathrm{FCFS}$ schedule. Here the forms are checked by the server and the people are divided into two categories according to 'years after marriage'. Clinic uses this as the initial predictor and does not check the other details in the first node. 'Less than one year after marriage' couples routed to the second node with probability $\mathrm{p}_{1}$. 'More than one year after marriage' couples can directly enter the third node for treatment with probability $1-\mathrm{p}_{1}$. In the former case, the details of the couples are checked and they undergo a counseling process. This node also follows an $M|M| 1|K| F C F ~ S$ schedule. After the counseling session, the couples who realized that they do not require treatment leave the system with probability $\mathrm{p}_{2}$. If they want treatment, directed to the third node with probability $1-\mathrm{p}_{2}$. This third node follows an $\mathrm{M}|\mathrm{M}| 1|\mathrm{~K}| \mathrm{F}$ CF S schedule. Here couples are treated and they leave the system with probability $\mathrm{p}_{3}$. This type of leaving may be due to completion of treatment (such as one day treatment) or giving up of treatment. Some couples want more cycles of treatments they re-enter the same node (immediate feedback) with probability $\mathrm{p}_{4}$. Some may be mentally stressed after consultation and want counseling and so can enter the second node with probability $1-p_{3}-p_{4}$ (delayed feedback). The queueing network envisaged here is that of three Markovian queueing models with a Bernoulli schedule for routing and with feedbacks.

\section{MODELING THE SERVICE SYSTEM}

Following are the notations used in this paper:

$\alpha=$ External Poisson arrival rate to the network

$\lambda_{\mathrm{j}}=$ Poisson arrival rate to node $\mathrm{j}$

$\lambda_{\text {ej }}=$ Effective arrival rate to node $\mathrm{j}$

$\mu_{\mathrm{j}}=$ Exponential mean service rate to node $\mathrm{j}$

$\mathrm{K}=$ Capacity of the node

$\mathrm{R}=$ Throughput rate

$\mathrm{h}_{\mathrm{ij}}=$ Holding node from node $\mathrm{i}$ to node $\mathrm{j}$

$\mu_{\mathrm{ej}}=$ Effective service rate at node $\mathrm{j}$ due to blocking

$\mathrm{P}_{\mathrm{K}}=$ Blocking probability of finite queue of size $\mathrm{K}$

$\mathrm{P}_{\mathrm{K}}{ }^{\prime}=$ Feedback blocking probability

$\mathrm{L}_{\mathrm{j}}{ }^{\mathrm{q}}=$ Average number of customers in the queue node $\mathrm{j}$

$\mathrm{W}_{\mathrm{j}}^{\mathrm{q}}=$ Average time a customer spends at node $\mathrm{j}$

We consider an open queueing network consisting of three single server nodes (service centers) and $Q_{i}$ denote the queue corresponding to the $\mathrm{i}^{\text {th }}$ node where $\mathrm{i}=1,2,3$. Customers arrive to the system according to a homogeneous Poisson process (external arrival) and get self service with a general service time distribution. After that customers are entered to the first node with rate $\alpha$. We assume that the service times are exponentially distributed with service rates $\mu_{1}, \mu_{2}$ and $\mu_{3}$ at the respective nodes. We also assume that there is an infinite waiting space capacity for each node, a single class of patients and that patients served on FCFS discipline. The routing probabilities in the network are assumed to be $\mathrm{p}_{1}$ from node one to node two, $\mathrm{p}_{2}$ is the leaving probability from node two and $1-\mathrm{p}_{2}$ is the transfer probability from node two to node three. $\mathrm{p}_{3}$ is the leaving probability from node three. Third node has immediate feedback and delayed feedback with probability $\mathrm{p}_{4}$ and $\left(1-p_{3}-p_{4}\right)$ respectively. Both types of feedbacks are assumed to be independent of the state of the system and any previous feedback. A diagrammatic representation of the above queueing network is shown in Figure 1. 


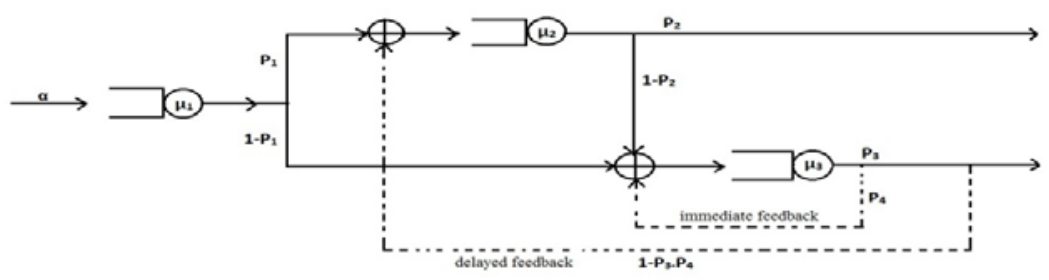

Figure 1 : Health care queueing network with 3 nodes and 2 feedbacks

\section{METHODOLOGY}

The waiting room restriction causes great difficulty in the analytical modeling of the network system because customers get blocked and the output distributions at some nodes are no longer identical. In order to analyze the network we use expansion methodology proposed by Kerbache and MacGregor Smith [18, 19] and decomposition approach. There is an immediate feedback at node three; immediate feedback corresponds to a service time equal to two consecutive service times. So for the further analysis we eliminate this immediate feedback at node three by adjusting the service times and the transition probability for customers leaving this node. After this reconfiguration we use decomposition method to partition the network into sub networks. Then we use expansion method to analyze each node individually. Using this method we expand the network by adding holding nodes. This transforms the network into an equivalent Jackson network where each node can be solved independently. After calculating the parameters of expanded network, throughput of the node is calculated. If the node is in tandem, after calculating the throughput of the first node it is used as the arrival rate of the second node. If node one is a split node, the arrival rate to the parallel node is the product of the throughput from the first node and transition probability to that node. If the second node is a merge node, arrival rate to the node is the sum of the throughputs of the arriving (previous) nodes. Similarly throughputs of all the nodes are calculated. Final node throughput becomes the entire throughput of the network.

In this network first node is a split node. The parallel nodes are two and three. Node three is a merge node. Considering feedback node two also acts as merge node. Customers leaving through two stations (nodes two and three). The throughput of the network is the sum of throughputs of nodes two and three.

\section{Analysis of the Network System}

Using the above methodology we find the throughput of the network and steps are as follows:

Step I: In the analysis of the network first step is to eliminate the immediate feedback of node three. In our model the transition probabilities are $\mathrm{p}_{33}=\mathrm{p}_{4}, \mathrm{p}_{30}=\mathrm{p}_{3}$ and $\mathrm{p}_{32}=\left(1-\mathrm{p}_{3}-\mathrm{p}_{4}\right)$. Using Bose [2], we eliminate the feedback by modifying the service rate and normalizing the transition probabilities as

$$
\begin{array}{rlrl}
\mu_{i} & =\mu_{i}\left(1-p_{i i}\right) & \\
p_{i j} & =\frac{p_{i j}}{\left(1-p_{i i}\right)} & & \text { for } i \neq j \\
& =0 & \text { for } i=j
\end{array}
$$

So the service rate and transition probabilities of the model becomes,

$$
\begin{aligned}
\mu_{3} & =\mu_{3}\left(1-p_{4}\right), \\
p_{30} & =\frac{p_{3}}{\left(1-p_{4}\right)}, \\
p_{32} & =\frac{\left(1-p_{3}-p_{4}\right)}{\left(1-p_{4}\right)} .
\end{aligned}
$$

Then after feedback elimination the network has the diagrammatic representation as shown in Figure 2. 


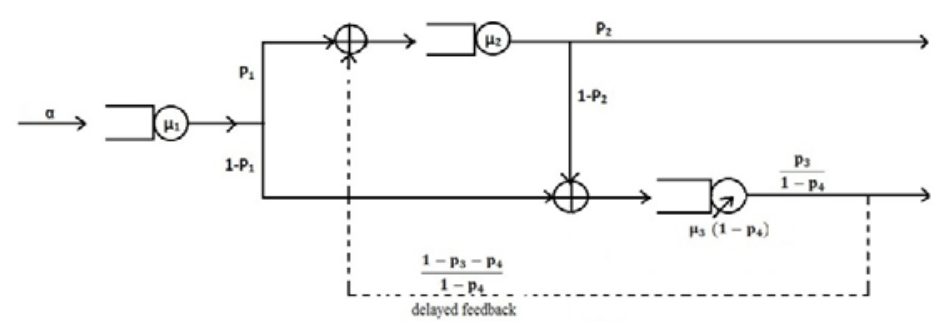

Figure 2: Healthcare queueing network after immediate feedback elimination

Step II: For the analysis network, next we decompose the network into two subnet-works as shown in Figure3.
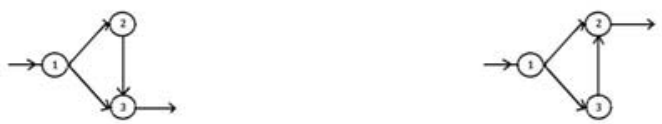

Figure 3: Split and merge topologies of the network

In Figure 3 the first sub network is a three node network with split and merges topology, node one split and node three is merge. The throughput of the third node is the sum of throughputs of one and two, which is the total throughput of the sub network. Similarly the second one is also a split and merge type, node one split and node two is merge. The throughput of the second node is the sum of throughputs of one and three, which is the total throughput of the sub network. Thus throughput of the whole network is the sum of throughputs of these sub networks.

Step III: After decomposing the network we use expansion method for further analysis. All the nodes are of finite capacity and external arrival to node one only. So we expand the sub networks by adding a holding node in front of each node (see Figure 4). These holding nodes follow the patterns of $\mathrm{M}|\mathrm{M}| \infty$ queue. If the customer arrive at the finite node, if the buffer is full; the customer entry will be stopped and is directly routed to the holding node with probability $\mathrm{p}_{\mathrm{K}}$. On the other hand, If the buffer is not full at the time of customer arrival, the job is routed to downstream node with probability $\left(1-p_{K}\right)$. Once the rejected customer enters the holding node, he stays there until a space becomes available at the previously rejected node. If the queue is still full, the customer is re routed to the holding node with probability $\mathrm{p}_{\mathrm{K}}{ }^{\prime}$. When a space available at the previously rejected node the customer immediately enters that node with probability $\left(1-\mathrm{p}_{\mathrm{K}}{ }^{\prime}\right)$. In the next stage we estimate all these parameters using the steps for parameter estimation (see Kerbache and MacGregor Smith [19]). Thus we calculate the throughput, the factor dependent on utilizations of the node and the preceding node of the whole network.

\section{Calculation of Parameters}

For each node $\mathrm{j}$ we determine $\mathrm{p}_{\mathrm{K}}, \mathrm{p}_{\mathrm{K}}{ }^{\prime}$ and $\mu_{\mathrm{h}}$ using the following equations ( see Kerbache and MacGregor Smith [19] ):

$$
\begin{gathered}
\lambda_{j}=\lambda_{i}-\lambda_{h_{j}}\left(1-p_{K}^{\prime}\right) . \\
\lambda_{i}=\lambda_{e i}\left(1-p_{K}\right)+\lambda_{h}\left(1-p_{K}^{\prime}\right) \\
\lambda_{h}=\lambda_{e i} p_{K} \\
p_{K}^{\prime}=\left[2-\frac{\lambda_{j}\left(r_{2}^{K}-r_{1}^{K}\right)-\left(r_{2}^{K-1}-r_{1}^{K-1}\right)}{\left.\mu_{h}\left(r_{2}^{K+1}-r_{1}^{K+1}\right)-\left(r_{2}^{K}-r_{1}^{K}\right)\right]^{-1}}\right. \\
r_{1_{j}}=\frac{\left[\left(\lambda_{j}+2 \mu_{j}\right)-\sqrt{z_{j}}\right]}{2 \mu_{j}}, \\
r_{2_{j}}=\frac{\left[\left(\lambda_{j}+2 \mu_{j}\right)+\sqrt{z_{j}}\right]}{2 \mu_{j}} \\
z_{j}=\left(\lambda_{j}+2 \mu_{j}\right)^{2}-4 \lambda_{j} \mu_{j} .
\end{gathered}
$$




$$
p_{K}=\frac{\left(1-\frac{\lambda_{e i}}{\mu_{h}}\right)\left(\frac{\lambda_{e i}}{\mu_{h}}\right)^{K}}{1-\left(\frac{\lambda_{e i}}{\mu_{h}}\right)^{K+1}} .
$$

Solving these equations we get the parameters corresponding to node $\mathrm{j}$. For the final throughput calculation,

$$
\begin{gathered}
R=\mu_{N}\left(1-P_{0}^{N}\right), \quad N=2,3 . \\
P_{0}{ }^{N}=\frac{\rho_{N}\left(1-\rho_{N}{ }^{K+1}\right)}{\left(1-\rho_{N}{ }^{K+2}\right)}
\end{gathered}
$$

where

$$
\rho_{N}=\frac{\lambda_{e N}}{\mu_{e N}}, \text { and } \lambda_{e N}=\left(1-p_{K}\right) \lambda_{N-1}
$$

In our model the throughput of the first node is $\alpha$, the external arrival rate. Then the node one split into two, so the arrival rates are product of routing probability to that node and external arrival rate $\alpha$. In the case of merge node arrival rate is the sum of throughputs to that node. In this case nodes two and three are departure nodes, the throughput of the network is the sum of throughputs of nodes two and three.

Using the following steps we calculate the throughput of the network:-

Step 0: Given values are external arrival rate $\alpha$, service rates $\mu_{\mathrm{j}}$, capacity of each node $\mathrm{K}, \mathrm{j}=1,2,3$.

Step 1: Throughput of the first node is $\alpha$, find $\mathrm{p}_{\mathrm{K}}$.

Step 2.0: If second node is the departure node, then couples enter this node by two ways, from node one and node three.

Step 2.1: Couples enter this node from node one with throughput $\alpha \mathrm{p}_{1}$.

Step 2.2: Find $\mathrm{p}_{\mathrm{K}}, \mathrm{p}_{\mathrm{K}}{ }^{\prime}$ and find the throughput.

Step 2.3: Couples also enter this node from third node, find throughput using step 3.1 and 3.2.

Step 2.4: Throughput of the sub network is the sum of throughputs of first and third nodes respectively. Calculate throughput rate $\mathrm{R}$.

Step 3.0: If third node is the departure node, customers enter this node by two ways, from node one and node two.

Step 3.1: Couples enter third node from node one with throughput $\alpha\left(1-\mathrm{p}_{1}\right)$.

Step 3.2: find $\mathrm{p}_{\mathrm{K}}, \mathrm{p}_{\mathrm{K}}{ }^{\prime}$ and find the throughput.

Step 3.3: Couples also enter third node from second node, and find throughput using step 2.1 and 2.2.

Step 3.4: Throughput of the third node is the sum of throughputs of first and second nodes respectively. Calculate throughput rate $\mathrm{R}$.

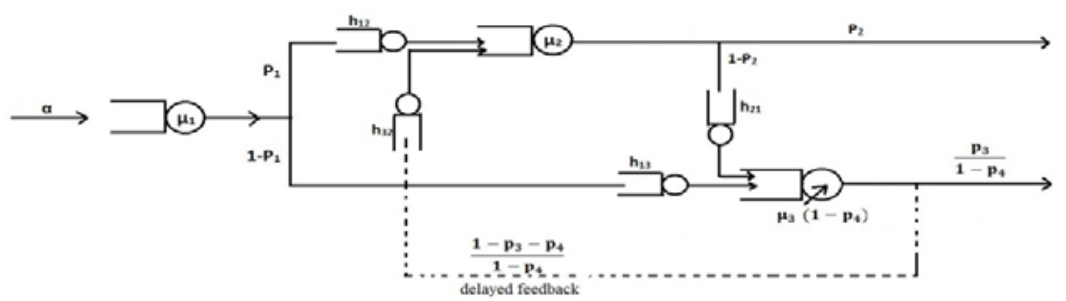

Figure 4 : Health care queueing network after expansion with holding nodes

\section{PERFORMANCE MEASURES}

The performance measures of each finite node can be obtained independently using the usual techniques available in literature. Our interest only in balanced case means $\rho_{j}<1$ (where $\rho_{j}$ is the ratio of effective arrival rate of node $\mathrm{j}\left(\lambda_{\mathrm{ej}}\right)$ and effective service rate of node $\left.\mathrm{j}\left(\mu_{\mathrm{ej}}\right), \mathrm{j}=1,2,3\right)$. In our model customers arrive according to Poisson process with rate $\alpha$. Assume that this node follows limited waiting space $\mathrm{K}$ including the one in service. A couple can join the network when there are less than K couples in the first node. If an arriving couple finds the node is full $(\mathrm{K})$, then the couple leaves the system. After service at the first node couples are 
directed to two nodes simultaneously. These two nodes are also follows finite capacity. So by the concept of holding node we expand path of the network with holding nodes in between nodes one and two, nodes two and three and three and two. The performance measures of each node can be calculated using the concept of effective arrival rate and service rate. So the performance measures corresponding to the network $(\rho<1)$ are the following.

\section{Expected Number in the Queue Node}

$$
L^{q}{ }_{j}=\frac{\rho_{j}}{\left(1-\rho_{j}\right)}-\frac{\rho_{j}\left(1+K \rho_{j}{ }^{K}\right)}{1-\rho_{j}^{K+1}}, \quad j=1,2,3 .
$$

\section{Expected Waiting time in the Queue Node}

$$
W^{q}{ }_{j}=\frac{1}{\mu_{j}}\left[\frac{\rho_{j}}{\left(1-\rho_{j}\right)}-\frac{K \rho_{j}^{K}}{1-\rho_{j}^{K}}\right], \quad j=1,2,3 .
$$

\section{Node One}

Performance measures of node one can be calculated using equations ( 1 and 2 ). External arrival rate is $\alpha$ so the effective arrival rate of node one $\left(\lambda_{1}\right)$ in this case is $\alpha$. There is no holding nodes before node one so the service rate is $\mu_{1}$ itself. Then $\rho_{1}$ can be calculated as $\rho_{1}=\left(\alpha / \mu_{1}\right)$. Using (1 and 2) we can find the average number and average waiting time of this queue node.

\section{Node Two}

Couples can enter this node in two ways. One from node one and other from node three. So because of the finite capacity there is a holding nodes in between nodes one and two and three and two. Queue length of node two is the sum of queue lengths from node one to node two and node three to node two. In this case is the effective arrival rates from node one to node two and node three to node two. Waiting time is also the sum of waiting times of these two paths. Effective service rates are calculated by

$$
\mu_{h}^{\prime}=\left(1-p_{K}^{\prime}\right) \mu_{h}
$$

And mean service time (reciprocal of the service rate) is

$$
\mu_{e i}^{-1}=\mu_{i}^{-1}+p_{K} \mu_{h}^{-1}
$$

Substituting the effective arrival rates and service rates we can calculate the average queue length and average waiting time respectively.

\section{Node Three}

The couples can enter this node in two ways. One way from node one and other from node two. Queue length and waiting times are the sum of their respective queue lengths and waiting times. The arrival rates and service rates are calculated same way as node two. Then substituting the arrival rates and service rates we can calculate the respective queue length and waiting time.

\section{NUMERICAL EVALUATION}

A Matlab program has been developed for the throughput analysis and the other performance measures. A simulation model was developed for $\mathrm{M}|\mathrm{M}| 1 \mid \mathrm{K}$ queueing net-works using MATLAB discrete event simulation modeling and its extension 'SimEvents'. Simulation experiments are necessary for these situations because of the unavailability of the actual data for the validation of the model. Throughput analysis while changing the arrival rates are studied under this model. The throughput presented was obtained by taking the average of 100 , 500 and 1000 simulation times. For different capacity of node throughput, average queue length and average wait are also studied using simulation model. The experiment was done by assigning routing probabilities, $\mathrm{p}_{1}=$ $0.4, \mathrm{p}_{2}=0.5, \mathrm{p}_{3}=0.4$ and $\mathrm{p}_{4}=0.3$ and the corresponding exponential service rates as $\mu_{1}=1.1, \mu_{2}=1.2$ and $\mu_{3}=$ 1.3. The experiment is repeated for external Poisson arrival rates $\lambda$ ranging from 0.1 to 0.9 with capacity $\mathrm{K}=2$. The throughput results of both analytical and simulation are presented in Tables 1-2. Table 3 gives the comparison result of arrival rate and queue length. The Table 4 illustrates the results of arrival rate vs. waiting time. 
Table 1: Throughput versus capacity when $\alpha=0.5$

\begin{tabular}{|c|c|c|c|c|}
\hline Capacity & \multicolumn{2}{|c|}{ Analytical } & \multicolumn{2}{c|}{ Simulation } \\
\hline & node2 & node 3 & node2 & node 3 \\
\hline 1 & 0.1820 & 0.2345 & 0.1910 & 0.2690 \\
\hline 2 & 0.1972 & 0.2445 & 0.2060 & 0.2790 \\
\hline 3 & 0.1995 & 0.2472 & 0.2130 & 0.2860 \\
\hline 4 & 0.1999 & 0.2479 & 0.2140 & 0.2870 \\
\hline 5 & 0.2000 & 0.2481 & 0.2150 & 0.2890 \\
\hline
\end{tabular}

Table 2: Summary of Throughput analysis $\mathrm{K}=2$

\begin{tabular}{|c|c|c|c|c|}
\hline $\begin{array}{c}\text { Arrival } \\
\text { rate }\end{array}$ & \multicolumn{2}{|c|}{ Analytical } & \multicolumn{2}{c|}{ Simulation } \\
\hline & node2 & node 3 & node2 & node 3 \\
\hline 0.1 & 0.0400 & 0.0496 & 0.036 & 0.0410 \\
\hline 0.2 & 0.0798 & 0.0992 & 0.072 & 0.1113 \\
\hline 0.3 & 0.1194 & 0.1484 & 0.122 & 0.1743 \\
\hline 0.4 & 0.1586 & 0.1969 & 0.159 & 0.2303 \\
\hline 0.5 & 0.1972 & 0.2445 & 0.206 & 0.2790 \\
\hline 0.6 & 0.2352 & 0.2907 & 0.235 & 0.3250 \\
\hline 0.7 & 0.2724 & 0.3352 & 0.262 & 0.3557 \\
\hline 0.8 & 0.3087 & 0.3778 & 0.277 & 0.3730 \\
\hline 0.9 & 0.3441 & 0.4182 & 0.299 & 0.3997 \\
\hline
\end{tabular}

Table 3: Arrival rate versus queue length

\begin{tabular}{|c|c|c|c|c|c|c|}
\hline $\begin{array}{c}\text { Arrival } \\
\text { rate }\end{array}$ & \multicolumn{3}{|c|}{ Analytical } & \multicolumn{3}{c|}{ Simulation } \\
\hline & node1 & node2 & node3 & node1 & node2 & node3 \\
\hline 0.1 & 0.0091 & 0.0013 & 0.0039 & 0.0047 & 0.0062 & 0.0064 \\
\hline 0.2 & 0.0404 & 0.0054 & 0.0166 & 0.0416 & 0.0137 & 0.0395 \\
\hline 0.3 & 0.1023 & 0.0126 & 0.0398 & 0.0970 & 0.0365 & 0.1288 \\
\hline 0.4 & 0.2076 & 0.0231 & 0.0756 & 0.1615 & 0.0546 & 0.2432 \\
\hline 0.5 & 0.3770 & 0.0375 & 0.1272 & 0.3811 & 0.1514 & 0.4152 \\
\hline
\end{tabular}

Table 4: Arrival rate versus waiting time

\begin{tabular}{|c|c|c|c|c|c|c|}
\hline $\begin{array}{c}\text { Arrival } \\
\text { rate }\end{array}$ & \multicolumn{3}{|c|}{ Analytical } & \multicolumn{3}{c|}{ Simulation } \\
\hline & node 1 & node2 & node3 & node1 & node2 & node3 \\
\hline 0.1 & 0.0909 & 0.0393 & 0.0777 & 0.0526 & 0.1010 & 0.0580 \\
\hline 0.2 & 0.2020 & 0.0809 & 0.1639 & 0.2174 & 0.1217 & 0.1619 \\
\hline 0.3 & 0.3409 & 0.1251 & 0.2601 & 0.3133 & 0.1870 & 0.3302 \\
\hline 0.4 & 0.5191 & 0.1721 & 0.3686 & 0.4069 & 0.2199 & 0.4829 \\
\hline 0.5 & 0.7542 & 0.2222 & 0.491 & 0.7486 & 0.4601 & 0.6694 \\
\hline
\end{tabular}

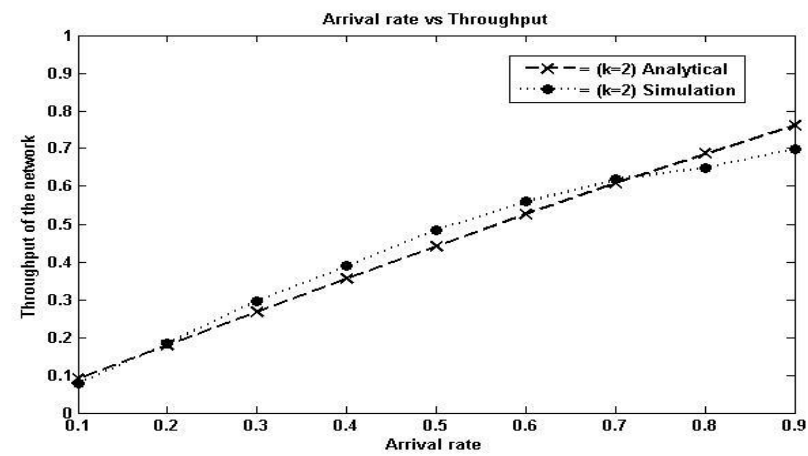

Figure 4 : Throughput of the network vs arrival rate 


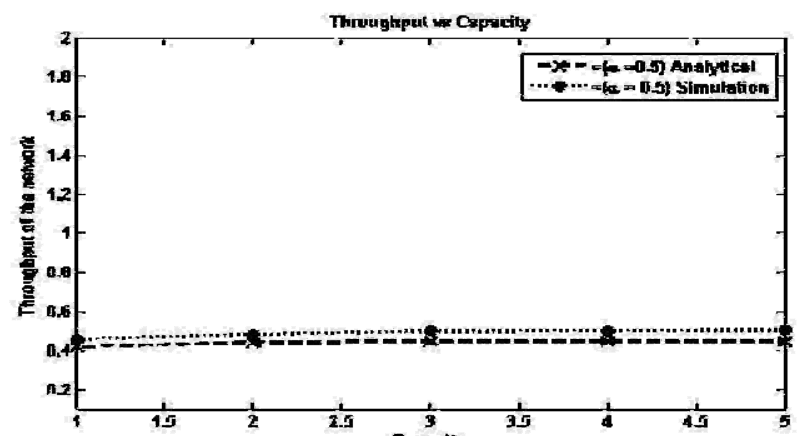

Figure 5 : Throughput of the network vs capacity

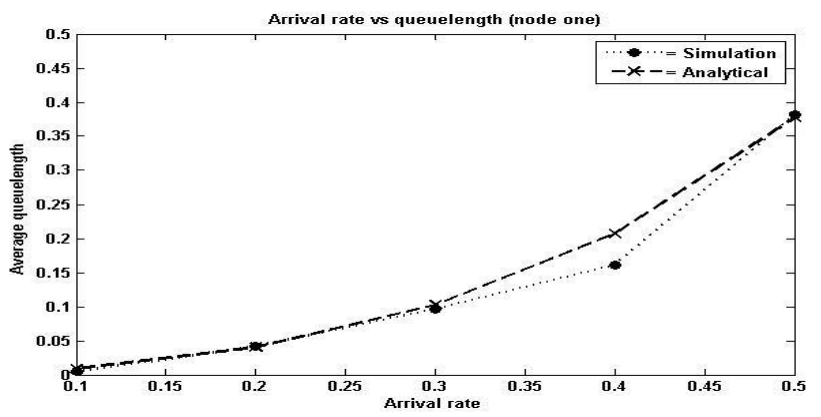

Figure 6 : Arrival rate vs queue length(node one)

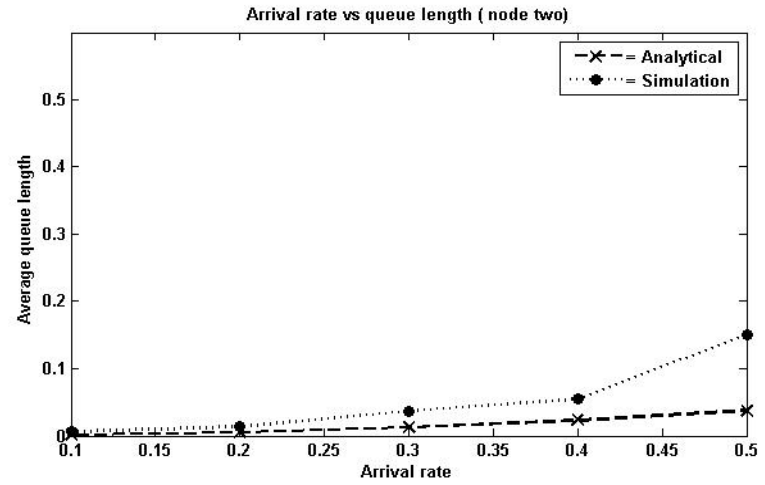

Figure 7: Arrival rate vs queue length (node two)

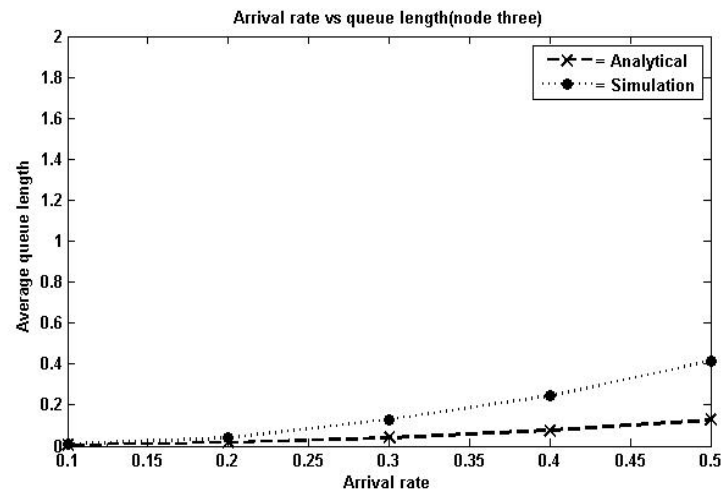

Figure 8: Arrival rate vs queue length (node three) 


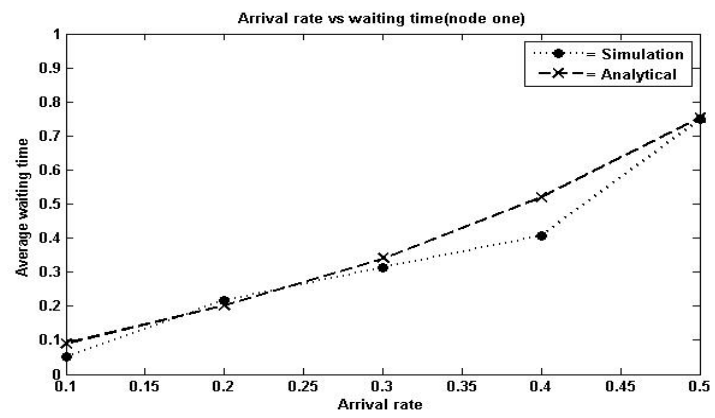

Figure 9: Arrival rate vs waiting time (node one)

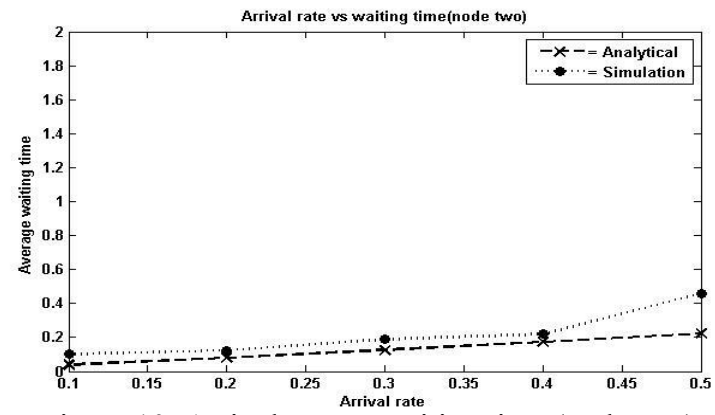

Figure 10: Arrival rate vs waiting time (node two)

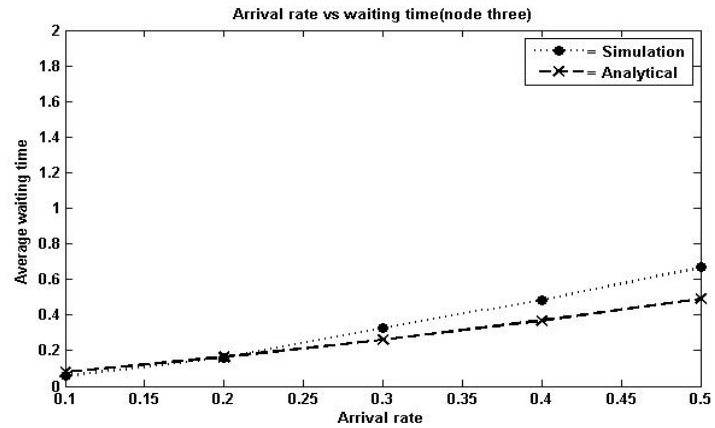

Figure 11: Arrival rate vs waiting time (node three)

Analytical and simulation results show that both arrival rate and capacity of the nodes increases throughput of the network also increases. Both simulation study and analytical study almost agree. Figure 4 illustrates the graph of the throughput of the network at different values of arrival rates. Figure 5 gives the graph of the throughput of the network vs capacity. Figures 6-8 gives the graph of comparison of arrival rate vs queue length. Similarly Figures 9-11 also gives the simulation and analytical study graphs of arrival rate vs waiting time.

\section{CONCLUSION}

In this paper we presented an approximation technique for the analysis of a finite open queueing network with transfer blocking and feedback. We address an arbitrary topology network with a special consideration of an infertility clinic health care system based on $\mathrm{M}|\mathrm{M}| 1 \mid \mathrm{K}$ model and then using expansion methodology the performance measures and throughput of each node are calculated. A MATLAB program was developed for the analysis of arbitrary topology finite open networks. A simulation model has been constructed using MATLAB Simulink extension 'SimEvents'. The numerical experiment was done using different parametric values and throughput and performance measures are calculated. In the comparison study it is revealed that the results of analytical and simulation do not differ significantly. So we conclude that the methodology suggested for the analysis of these types of networks is quite feasible and can be used for any general topology feedback networks. 


\section{REFERENCES}

[1] N.T.J. Bailey, A study of queues and appointment systems in hospital out-patient departments with special reference to waiting-times, J. Roy. Statist. Soc. Ser. B 14(2) (1952) 185-199.

[2] S.K. Bose, An introduction to queueing Systems, Kluwer academic / Plenum publishers, New York, 2002.

[3] P.J. Burke, The output process of a stationary M|M|s queueing system, Ann. Math. Stat. 39 (1968) 1144-1152.

[4] M. Carter, Diagnosis: Mismanagement of resources, OR/MS Today 29(2) (2002) 26-32.

[5] F.R.B. Cruz and T.V. Woensel, Finite queueing modeling and optimization: A selected review, Journal of Applied Mathematics. Article ID 374962, (2014) 1-11.

[6] A.A. Fredericks, Congestion in blocking systems a simple approximation technique, BellSys. Tech. J. 59 (1980) 805-827.

[7] J.A. Goddard, M. Malek, M. Tavakoli, An economic model of the market for hospital treatment for non-urgent conditions, Health Econ. 4(1) (1995) 41-55.

[8] E. Gelenbe and G. Pulolle, The behaviour of a single queue in a general queueing network, ACTAINF 7 (1976) 123-136.

[9] R.W. Hall, Patient flow: Reducing delay in health care delivery, Springer, New York, 2006a.

[10] R.W. Hall, Patient flow: The new queueing theory for health care, OR/MS Today 23(3) (2006b) 36-40.

[11] P.R. Harper, A.K. Shahani, Modeling for the planning and management of bed capacities in hospitals, J. Oper. Res. Soc. 53(1) (2002) 11-18.

.C. Hershey, E.N. Weiss, M.A. Cohen, A stochastic service network model with application to hospital facilities, Oper. Res. 29(1) (1981) $1-22$

.R. Jackson, Job shop-like queueing systems, Manag. Sci. 10 (1963) 131-142.

.R. Jackson, Networks of waiting lines, Oper. Res. 5(4) (1957) 518-521.

[15]

.R.P. Jackson, J.D. Welch, J. Fry, Appointment systems in hospitals and general practice, J. Oper. Res. Soc. 15(3) (1964) 219-237.

[16]

. Jain and J.M. Smith, Open Finite queueing networks with M/M/C/K parallel servers, Computers and Operations Research. 21(3) (1994) 297-317.

. Kavusturucu and S.M. Gupta, Expansion method for the throughput analysis of open finite manufacturing/ queueing networks with N-policy, Computers and Operations Research. 26 (1999) 1267-1292.

. Kerbache and J.M. Smith, The generalized expansion method for open finite queueing networks. European Journal of Operational Research. 32 (1987) 448-461.

. Kerbache and J. MacGregor Smith, Asymptotic behaviour of the expansion method for open finite queueing networks, Computers. Ops. Res. 15(2) (1988) 157-169.

Koizumi, Modeling patients flows using a queueing network with blocking, Health Care Manag. Sci. 8 (2005) 49-60

$\mathrm{N}$

J. Kuehn, Approximate analysis of general queueing networks by decomposition, IEEE Transactions on communications, 27(1) (1979) 113-126.

[22] J. Labetoulle, G. Pujolle, Isolation method in a network of queues, IEEE Trans.Softw.Engng SE- 5(5) (1979) 530-538.

[23] N.M. Mirasol, The output of an $M|G| \infty$ queueing system is Poisson, Proc. Camb. Phil. Soc. 72 (1963) 137-169.

[24] B. Melamed, Characterization of Poisson traffic system in Jackson queueing net-works, Adv. Appl. Prob. 11 (1979) 422-438.

[25] H.G. Perros, Queueing networks with blocking: a bibliography, ACM Sigmet. 12 (1984) 8-12.

[26] M. Reiser and H. Kobayashi, Accuracy of the diffusion approximation for some queueing systems, Journal of Research and development. 18 (1974) 110-124.

[27] Y. Roumani, Modeling patient flow in a network of Intensive Care Units (ICUs), Doctoral dissertation, University of Pennsylvania, 2013.

[28] A.J. Singh and J.M. Smith, Buffer allocation for an integer nonlinear network design problem, Computers and Operations Research, 24(5) (1997) 453-472.

[29] M.S. Sreekala and M. Manoharan, Analysis of restricted queueing networks- a blocking approach, 2016 (unpublished).

[30] B.W. Taylor III, A.J. Keown, A network analysis of an inpatient/outpatient department, J. Opl. Res. Soc. 31(2) (1980) 169-179.

[31] D. Worthington, Hospital waiting list management models, J. Opl. Res. Soc. 42(10) (1991) 833-843.

[32] F. Zegers-Hochschild, G.D. Adamson, J. de Mouzon, O. Ishihara, R. Mansour, K. Nygren, E. Sullivan, S. Vanderpoel, International Committee for Monitoring Assisted Reproductive Technology (ICMART) and the World Health Organization (WHO) revised glossary of ART terminology, Fertil. Steril. 92(5) (2009), 1520-1524. 